\title{
Dosimetric and clinical factors predicting quality of life after post prostatectomy radiation therapy: useful tool or not?
}

\author{
Christian Carrie $^{1}$, Gabriel Bringeon ${ }^{2}$ \\ ${ }^{1}$ Department of Radiation Oncology, Centre Leon Berard, Lyon, France; ${ }^{2}$ Department of Urology, Infirmerie Prostestante, ${ }^{3}$ Chemin du Penthod, \\ Caluire et Cuire, France \\ Correspondence to: Christian Carrie, MD. Department of Radiation Oncology, Centre Leon Berard, 28 rue Laennec 69008, Lyon, France. \\ Email: christian.carrie@lyon.unicancer.fr. \\ Provenance: This is an invited article commissioned by Section Editor Xiao Li (Department of Urology, Jiangsu Cancer Hospital \& Jiangsu Institute \\ of Cancer Research \& Nanjing Medical University Affiliated Cancer Hospital, Nanjing, China). \\ Comment on: Akthar AS, Liao C, Eggener SE, et al. Patient-reported Outcomes and Late Toxicity After Postprostatectomy Intensity-modulated \\ Radiation Therapy. Eur Urol 2019. [Epub ahead of print].
}

Submitted Jul 19, 2019. Accepted for publication Jul 31, 2019.

doi: $10.21037 /$ tau. 2019.08 .04

View this article at: http://dx.doi.org/10.21037/tau.2019.08.04

Among all therapeutic alternatives for prostate cancer, radical prostatectomy $(\mathrm{RP})$ is one of the most frequent used especially in young patients. However, after RP one third of patients will relapse either with only a rising prostate specific antigen (PSA) or a persistent elevated post-surgery PSA.

We had the opportunity to read an interesting paper recently published in the EAU fournal by Akthar et al. (1). This article purpose is to guide the patients on what symptoms to expect with salvage radiotherapy (SRT) after $\mathrm{RP}$ and eventually a tool for physicians to modify clinical and radiation related risk factors in order to optimize quality of life (QoL). This prospective study reports 199 patients treated either with SRT alone or combined with androgen deprivation (ADT). Two third received a pelvic nodes irradiation, the tumor bed dose was $68 \mathrm{~Gy}$. The mean age of the population was quite low (63 years), combined with the fact that $99 \%$ of the 128 patients receiving pelvic irradiation also received $\mathrm{ADT}$ and that only $8 \%$ received SRT as adjuvant treatment, we can assume that at least two third of patients had locally advanced tumors and probably more likely to have a recurrence and therefore combined post surgery and post radiotherapy toxicities. The time to SRT after surgery is very short (19 months) probably due to early relapses for locally advanced disease or persistent elevated PSA after surgery.

The outcome measurements were done with the minimal clinically important difference (MCID) which is the smallest change in a treatment outcome that an individual patient would identify as important and which would indicate a change in the patient's management (2). Five QoL domains were explored: urinary irritation or obstruction (UI/UO), urinary continence (UC), overall urinary (UF), bowel (BF) and sexual function (SF). The median follow-up is only 33 months: too short for late effect but sufficient to at least evaluate acute toxicities except that only $27 \%$ providing patients reported outcome at 5 -year and $10 \%$ at 7 -year.

QoL remained stable for the 5 domains with no decline exceeding the MCID. However, it is a concern that the QoL for UC was stable but started with a low score $(60 \%)$ and moreover $82 \%$ patients needed pads at 2 months and 10 patients among the 20 evaluable patients at 84 months, reflecting probably the initial surgical difficulties for large tumors. It is also a concern that QoL is not related and do not change over time with the number of pads per day suggesting no decrease of continence during and after SRT (authors suggest even a recovery).

Not surprisingly sexual score is the worst but at 7 years only 20 patients were assessable. Two thirds have a sexual activity with sexual aid (PDE inhibitor). Since $66 \%$ of patients have had ADT for 4 to 48 months, we can assume that neither short ADT nor moderate irradiation dose jeopardized future sexual activity if present after the surgery.

Bladder V70 Gy was the only dosimetric parameter found as related with Gr2+ GU toxicities but probably marginally since the median dose to pelvic area was $50 \mathrm{~Gy}$ 
and to the prostate bed only 68 Gy. Among other factors, body mass index and age were also correlated with higher risk of impairment of QoL.

Finally, the study showed a favorable long-term QoL and few late toxicities after SRT with a small transient decline in the 5 domains. These results can be discussed with the other prospective series on SRT even if the QoL evaluation was not the primary end point.

Two recent randomized trials defined SRT with short or long androgen (deprivation treatment) as a standard in such patients $(3,4)$. In these two large trials the acute toxicities rate was less than $11 \%$ for grade 2 or more for acute genitourinary adverse events. Regarding late toxicities, genitourinary grade 3 occurred in $7 \%$ for the two trials. The QoL study score done in the Getug 16 (with QLQ-C30 global QoL score) showed no change the first year in half of patients, an improvement in $20 \%$ and worse in only $30 \%$ and the score was exactly the same 5 years later. In the Getug 16 trial the acute toxicities rate is less than $1 \%$ for sexual disorders and only $41 \%$ of patients had a sexual activity before salvage RT but the score dropped to $50 \%$ for patients without concomitant ADT 1 year after SRT $(25 \%$ for those having the combined arm SRT + ADT). Sexuality was not reported in the RTOG trial but most of toxicities were related to the long ADT.

It is important to note that these two trials have been conducted before the large use of intensity modulated radiotherapy (IMRT) which allows now a dramatic decrease of acute and late toxicities and that at least for Getug 16 trial the population had a more favorable stage at time of surgery.

These three papers, and more precisely the Akhtar's publication, confirm the relative safety of SRT after RP with less than $7 \%$ of $\mathrm{Gr} 2+$ of GI/GU toxicities and no increase of toxicities with time. The EAU study strength is the prospective evaluation of detailed QoL and the limits are the short $\mathrm{FU}$ and the high number of follow-up loss.

However, the three papers do not address exactly to the same population: Getug 16 had older patients (67 years old) but a high percentage of initially low stage patients (54\% stage 2 or below) explaining probably a long delay between surgery and recurrence at 2.5 years and only $15 \%$ of patients received a pelvic irradiation. The RTOG $9601 \mathrm{had}$ a mean age population of 65 , higher initial stage with $66 \%$ of stage 3 and a mix of through rising PSA and persistent PSA after surgery explaining a delay of 1.4 years between surgery and relapse. Most of the patients in the publication of Boston had a higher initial stage with $69 \%$ of stage T3a or T3b. Due this young age, the trend was probably to be more aggressive surgically with as consequences a short delay between surgery and relapse (19 months) and the risk to cumulate sequelae of surgery and SRT. Young age of patient is not enough to decide surgery for locally advanced disease if complete resection with negative margin is not reasonably achievable and careful pretreatment evaluation of QoL must be done before any decision.

\section{Acknowledgments}

None.

\section{Footnote}

Conflicts of Interest: The authors have no conflicts of interest to declare.

Etbical Statement: The authors are accountable for all aspects of the work in ensuring that questions related to the accuracy or integrity of any part of the work are appropriately investigated and resolved.

\section{References}

1. Akthar AS, Liao C, Eggener SE, et al. Patient-reported Outcomes and Late Toxicity After Postprostatectomy Intensity-modulated Radiation Therapy. Eur Urol 2019. [Epub ahead of print].

2. Cook CE. Clinimetrics Corner: The Minimal Clinically Important Change Score (MCID): A Necessary Pretense. J Man Manip Ther 2008;16:E82-3.

3. Carrie C, Dussart S. Salvage radiotherapy for patients with rising PSA - Authors' reply. Lancet Oncol 2016;17:e315.

4. Shipley WU, Seiferheld W, Lukka HR et al. Radiation with or without Antiandrogen Therapy in Recurrent Prostate Cancer. N Engl J Med 2017;376:417-28.

Cite this article as: Carrie C, Bringeon G. Dosimetric and clinical factors predicting quality of life after post prostatectomy radiation therapy: useful tool or not? Transl Androl Urol 2019;8(6):770-771. doi: 10.21037/tau.2019.08.04 